Appl. Phys. B 49, 239-244 (1989)

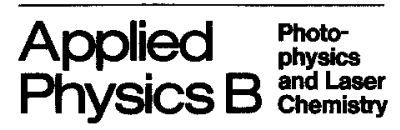

(C) Springer-Verlag 1989

\title{
KrF Laser System with Corrected Pulse Front and Compressed Pulse Duration *
}

\author{
S. Szatmári ${ }^{\star \star}$, G. Kühnle, J. Jasny ${ }^{\star \star \star}$, and F. P. Schäfer \\ Max-Planck-Institut für biophysikalische Chemie, Abteilung Laserphysik, \\ D-3400 Göttingen, Fed. Rep. Germany
}

Received 21 March 1989/Accepted 8 Juni 1989

\begin{abstract}
A high-power $\mathrm{KrF}$ laser system is presented providing $45 \mathrm{~mJ}, 150 \mathrm{fs}$ pulses at the position of a target. Pulse front distortion is avoided by specially designed refractive optics. Pulse compression is done after final amplification using a simplified compressor.
\end{abstract}

PACS: $42.60,42.55 \mathrm{M}$

High-power excimer laser pulses are of increasing interest for use in plasma physics and X-ray laser research. $\mathrm{KrF}$ lasers are superior to the other excimer lasers due to their short wavelength $(248 \mathrm{~nm})$, large and unmodulated gain bandwidth, allowing the amplification of pulses shorter than $100 \mathrm{fs}[1,2]$, and their highenergy extraction efficiency when operated as short pulse amplifiers [3, 4].

Short KrF laser pulses are generally obtained by amplification of frequency converted short dye-laser pulses in subsequent $\mathrm{KrF}$ amplifiers. The experimental realizations mainly differ in the way how the short pulses are generated and in the output pulse energy. The commonly used technique to obtain subpicosecond seed pulses at $248 \mathrm{~nm}$ is frequency mixing or tripling of pulses from mode-locked lasers, as first demonstrated in [5] and later by several other groups [6-9]. Such a system is quite complex and needs several lasers that must work together properly. We have developed an alternative method based on the use of a simple hybrid excimer-dye laser arrangement $[1,2]$. With this system and by additional pulse compression, pulses as short as $60 \mathrm{fs}$ were obtained at $248 \mathrm{~nm}$ [2].

* Dedicated to Prof. Dr. Herbert Welling on the occasion of his 60 th birthday

Permanent addresses: $\star \star$ JATE University, Research Group on Laser Physics of the Hungarian Academy of Sciences, Dóm tér 9, H-6720 Szeged, Hungary

$\star \star \star$ Polska Akademia Nauk, Instytut Chemii Fizycznej, Kasprzaka 44/52, PL-01-224 Warszawa, Poland
In this paper we present an extension of this system to higher pulse energies, at the expense of a minor increase in pulse duration. The two crucial components of the extended system are: first, correction of pulse front distortion by a special refractive optics, second, pulse compression after final amplification.

\section{Basic System}

The experimental arrangement is shown in Fig. 1a. It consists of a double-discharge excimer laser (EMG 150, Lambda Physik), whose oscillator channel
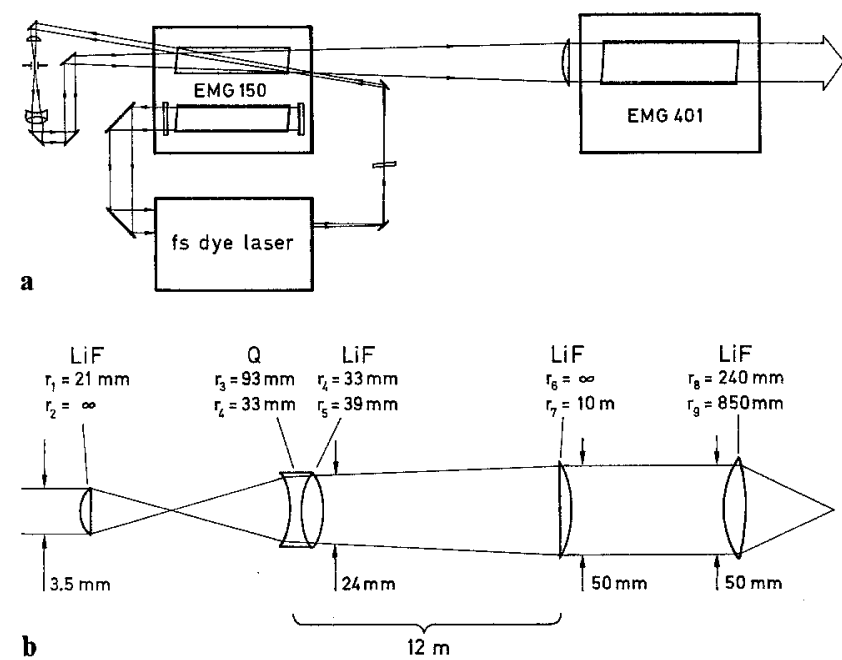

Fig. 1a, b. Schematic of the a experimental arrangement, b pulse front corrected UV optics 
is used to pump the subpicosecond dye laser amplifier arrangement described in [2]. The output pulses of the dye laser are frequency-doubled and then amplified in a double-pass arrangement in the amplifier channel of the EMG 150. Subsequently they are further amplified in a wide aperture excimer gain modul (EMG 401, Lambda Physik). The detailed description of the dye laser system has been given in $[2,10,11]$.

\section{Timing of the Seed Pulse}

The slightly divergent seed pulse of approximately $5 \mu \mathrm{J}$ leaving the frequency doubler is amplified in a first pass through the amplifier channel of the EMG 150, which is filled with $\mathrm{KrF}$ at a partial pressure of $6 \mathrm{mbar}$ of $\mathrm{F}_{2}$, $150 \mathrm{mbar}$ of $\mathrm{Kr}$ and with $\mathrm{He}$ to a total pressure of 2.5-3.0 bar. The total pressure is adjusted to shift the seed pulse in time to the position, when the gain starts rising, in order to get a good trade-off between sufficient amplification of the pulse and a minimum of amplified spontaneous emission (ASE) level before the pulse arrives. Increase of the pressure delays the gain rise, resulting in less ASE before the pulse, albeit at the expense of a decrease of amplification and a concomitant slight decrease in output energy (see below). The fact that the two discharge channels of the EMG 150 are switched by a single thyratron makes it possible to position the seed pulse with an accuracy of $\sim 100 \mathrm{ps}$ at the steep rise of the gain, and then to optimize the amplifier for the best compromise of highest output energy and least ASE.

\section{Beam Expansion Using Overcompensated Optics}

After the first pass the beam is magnified by a beam expanding telescope $(M \approx 7)$ and spatially filtered by a quartz pinhole $(\phi=10 \mu \mathrm{m})$ placed in an evacuated chamber at the focus of the telescope. The optical layout of the UV amplification is shown in Fig. $1 \mathrm{~b}$.

The first lens of the beam-expanding telescope between the two passes in the EMG 150 amplifier is a planconvex lens made of LiF. The second lens is an "overcorrected" achromat - made of LiF and quartz which compensates the pulse front distortion of the first lens and the following two lenses (Fig. 1b) made also of LiF. The radius of the different surfaces of the lenses is also indicated in Fig. 1b. This telescope is adjusted to give a slightly divergent beam in the second pass of the EMG 150 amplifier. After $12 \mathrm{~m}$ travel this beam has expanded sufficiently to fill the aperture of the collimator lens at the entrance part of the EMG 401 amplifier. This collimator lens is a $f=24 \mathrm{~m}$ planoconvex $\mathrm{LiF}$ lens, which is needed to make the beam parallel. The optics is made mainly of $\mathrm{LiF}$ to minimize nonlinear absorption [12] and designed to give no pulse front distortion [13] at the target after the focussing lens behind the EMG 401. The EMG 401 amplifier is filled with $4.5 \mathrm{mbar} \mathrm{F}_{2}, 100 \mathrm{mbar} \mathrm{Kr}$, and 3.5 bar Ne for optimum output energy and stability. The output energy measured after the amplifier is $65 \mathrm{~mJ}$, with $10 \%$ ASE content.

\section{Pulse Compression and Pulse Duration Measurements}

It was shown in $[1,2]$ that pulse compression of the output pulses of this $\mathrm{KrF}$ system always results in a 5-10 times reduction in the pulse width. In those experiments pulse compression and pulse duration measurements were performed after the second pass of the EMG 150 excimer amplifier, either using a singlepass [1] or a double-pass [2] double-prism pulse compressor. It was found that the duration of pulses compressed by a double pass compressor is always shorter than that obtained by single-pass compression. This result is obtained even with blocking the margins of the beam which contain only part of the pulse spectrum. This observation can be understood when considering a possible distortion in the pulse front [13] at the position of the single-pass pulse compressor. On the other hand, the use of single pass compression is advantageous to minimize losses.

From the point of view of optical losses, pulse compression would be ideal to be done before the last amplifier. For this reason we have built a double pass double prism pulse compressor, consisting of two $60^{\circ}$ quartz prisms, between the EMG 150 and EMG 401 lasers. (The construction of the pulse compressor is the same as described in [2].)

The pulse duration was measured after the EMG 401, applying the usual autocorrelation technique based on two-photon ionization of NO gas. The Michelson interferometer (Fig. 2a) is a modified version of that used in [2], optimized for high-power applications. This arrangement minimizes the lightpath length in optical materials, while allowing similar conditions for the two partial beams in the two arms of the interferometer. In our experiments a $5 \mathrm{~mm}$ thick uncoated $\mathrm{CaF}_{2}$ plate is used as a beam splitter, using the Fresnel reflection at the surfaces. Partial beams of each arm are once reflected from and transmitted through the $\mathrm{CaF}_{2}$ plate and combined at the signal output. Complete spatial overlap of the beams is accomplished by translation of the retroreflector of one arm in a direction perpendicular to the beam. The main beam (which is transmitted twice) is used as a reference. The size of the aperture at the input is chosen so that the beams created by the second reflection from the beamsplitter (shown as dashed lines in Fig. 2a) can be blocked by a beam stop (see also [15]). Then the 


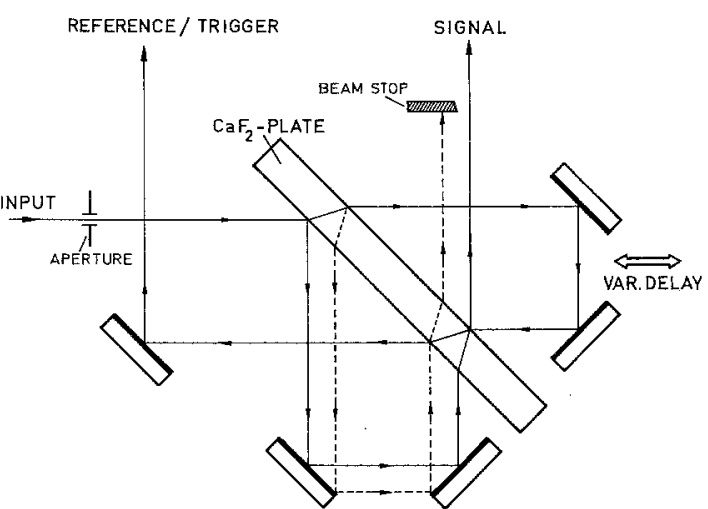

a

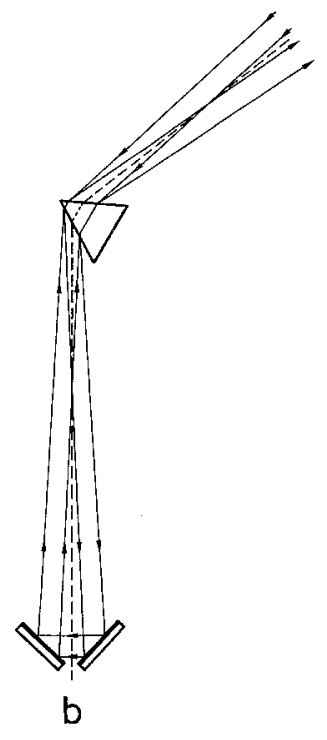

Fig. 2. a Autocorrelator used for pulse duration measurements, b schematic of the pulse compressor signal and the reference output are directed into two identical ionization chambers containing 100 Torr $\mathrm{NO}$, and the ratio of the ion currents is recorded as a function of the delay of the movable arm of the interferometer.

A set of autocorrelation functions were recorded after the EMG 401 with different settings of the pulse compressor placed between the two amplifiers. An optimum was found at a prism separation of $L=60 \mathrm{~cm}$, corresponding to a negative group velocity dispersion of $660 \mathrm{fs} / \mathrm{nm}$. Such an autocorrelation curve is shown in Fig. 3a, indicating $140 \mathrm{fs}$ autocorrelation width, when the EMG 401 amplifier is not in operation. The


Fig. 3a, b. Aurocorrelation curves of the output pulses when compression is done before the last amplifier. a The last amplifier is switched off, $\mathbf{b}$ in operation needed longer separation ( $L$ ) of the prisms of the compressor compared to [2], is the result of the increased group velocity dispersion of the system caused mainly by the two $10 \mathrm{~mm}$ thick $\mathrm{CaF}_{2}$ windows of the EMG 401 amplifier.

Within the accuracy of the measurement no temporal wings are observable in the autocorrelation curve of Fig. 3a. However, when switching on the EMG 401 amplifier, a strong increase of the autocorrelation width occurs, as seen in Fig. 3b. This increase of the pulse width could not be compensated by a different setting of the pulse compressor and hence is not due to some additional group velocity dispersion, but caused by a pulse-forming process in the excimer amplifier. It is known that nonlinearities in the chirp always appear as "uncompressable" temporal wings in the autocorrelation function of the compressed pulse. Even assuming relatively small-amplitude temporal wings, i.e. a temporal structure before the compressed main pulse, these can effectively be amplified in a $\mathrm{KrF}$ amplifier having a small-signal gain in the range of $10^{4}$ [3]. Due to saturation of the amplifier by the compressed main pulse, amplification for the main pulse and the trailing component decreases, which enhances the relative amplitude of the leading temporal wings to a level comparable to the main pulse, resulting in significant temporal broadening.

This is a clear indication that pulse compression should be done after the last amplifier. For this reason we developed a high efficiency pulse compressor, consisting of one $\mathrm{MgF}_{2}$ Brewster prism (Fig. 2b). In the principle of operation, this arrangement is similar to that of the single-pass double-prism compressor, but here a single-prism double-pass compressor is set up with the help of a retroreflector. The two beams are set symmetrically about the beam paths of minimum 



Fig. 4a, b. Autocorrelation curves when compression is done after amplification. a The last amplifier is switched off, $b$ in operation

deviation indicated as dashed line in Fig. 2b. The optical axis of the $60 \times 60 \times 70 \mathrm{~mm}^{3}$ prism (Dr. Karl Korth, Kiel) was chosen to be parallel to the base and perpendicular to the refractive edge of the prism within an accuracy of less than $3^{\circ}$ to minimize birefringence. The prism was positioned to minimize the pathlength in the prism, in order to minimize the positive group velocity dispersion, self phase modulation, and nonlinear absorption in the material. Even with the use of this arrangement the transmission of the compressor was $\sim 70 \%$. Two autocorrelation curves measured after this pulse compressor at optimum compensation, when the distance between the retroreflector and the prism $(\mathrm{L})$ is $150 \mathrm{~cm}$, are shown in Fig. 4a, when the EMG 401 amplifier is in operation, and in Fig. 4b, when the amplifier is switched off. In both cases an autocorrelation width of $\sim 230 \mathrm{fs}$, corresponding to $\sim 150$ fs pulse width - assuming $\operatorname{sech}^{2}$ pulse shape - is found. It is seen that the pulse width is independent of the operation of the last amplifier. However, the shortest pulse duration obtained with this arrangement is factor of 1.5 times longer than that obtained with double-pass pulse compression. To our knowledge this is partly due to the spatial displacement of the different spectral components in single-pass pulse compressors. If there were no curvature of the pulse front, it would suffice to block the margins of the beam where not all spectral components are present, as it was done here. If, however, there is a pulse front curvature of the uncompressed pulse in the form of a paraboloid, it will be deformed so that for a single spectral component the pulse front has still such a shape, but now the individual paraboloids are shifted with respect to each other, with a maximum possible shift $s$ between the longest and shortest wavelength components.

A simple estimation for this temporal broadening can be given as follows: If the uncompressed pulse front is distorted by the effect of lenses the temporal distribution of the pulse front in a parallel beam is given by the equation

$T=\alpha h^{2}$,

where $T$ is time, $\alpha$ is the second order coefficient of the distortion, $h$ is the distance measured from the centre of the axis of the rotational paraboloid of the pulse front [13]. If in the process of pulse compression the maximum shift $s$ as explained occurs the effective broadening is described by the equation

$\Delta T=2 \alpha h s$.

With a beam diameter $D$

$0 \leqq h \leqq\left|\frac{D-s}{2}\right|$

and $0 \leqq s \leqq D$ must be fulfilled.

For our experimental conditions $\alpha=0.23 \mathrm{fs} / \mathrm{mm}^{2}$ (see below), $s=5 \mathrm{~mm}$, and a worst case value of $h$ is $h=10 \mathrm{~mm}$. Then

$\Delta T=23 \mathrm{fs}$.

This broadening, obtained even with a pessimistic assumption of $h$ is less than the experimentally observed temporal broadening. The main reason is very likely related to a nonlinear chirp created by the $\mathrm{MgF}_{2}$ prism where the intensity is $\sim 30 \mathrm{GW} / \mathrm{cm}^{2}$ and/or the effect of residual birefringence resulting in an "uncompressable" temporal broadening.

\section{Pulse-Front-Curvature Measurements}

As described Sect. 1, the whole system, including the focussing optics, is designed to have minimum pulse front distortion just at the target. For technical reasons the pulse front distortion was not measured there, where the beam is convergent. Actually, the measurements for the pulse front were carried out before the focussing lens in a position where the beam is parallel, and expected to have some pulse front distortion due to the necessary precompensation of the effect of the focussing lens.

The interferometer used for this measurement is shown in Fig. 5. This is a modified version of that used in [13], capable of measuring pulse front distortions over several $\mathrm{cm}$, without having long path lengths in the beam splitters [16]. The arrangement consists of two slightly wedged uncoated quartz plates, which act as beam splitters, to split (beam splitter I), and to 


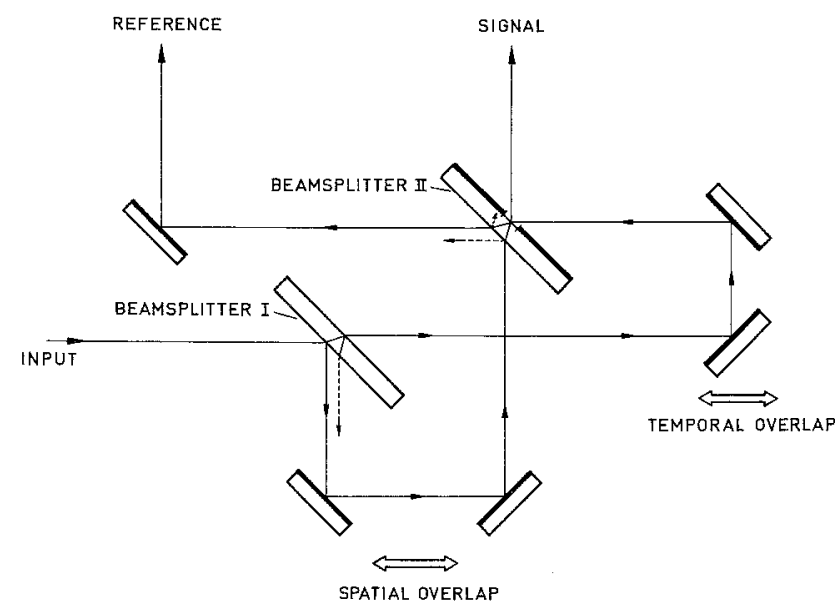

Fig. 5. Autocorrelator for the measurement of the pulse front distortion

recombine (beam splitter II) the two partial beams of the interferometer. The aperture put on the active surface of beam splitter II is positioned to transmit the middle part of the beam coming from the upper retroreflector. While the position of this partial beam is fixed at the aperture, the other can be shifted by shifting the lower retroreflector in the direction perpendicular to the beam. Therefore different parts of the input beam can be combined behind the aperture, where the autocorrelation measurement is done. The temporal overlap between the two beams can be adjusted by translating the upper retroreflector in the direction indicated in Fig. 5.

When the position of this retroreflector corresponding to complete temporal overlap (i.e., the peak of the autocorrelation curve) is recorded as a function of the shift of the lower retroreflector the curvature of the pulse front can be determined. Figure 6 shows the result of such a measurement when using the compressed output of the EMG 401. The horizontal scale is the displacement of one partial beam, the vertical one is the time of complete overlap between the two beams

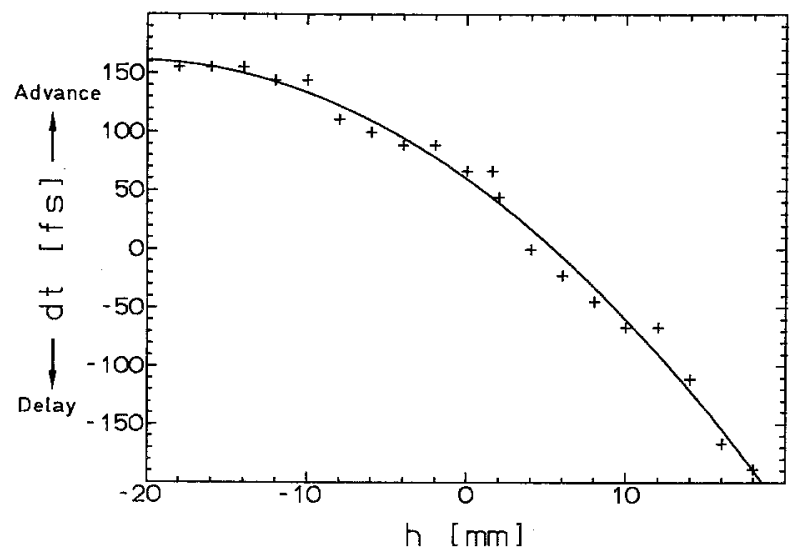

Fig. 6. Spatial dependence of the pulse front of the output pulses (time zero is set arbitrarily). One can see that the experimental points are not lying along a straight line, but can be fitted by a parabola whose square coefficient is

$\alpha=0.23 \pm 0.02 \mathrm{fs} / \mathrm{mm}^{2}$.

The linear part is caused by the not quite perpendicular linear translation of the retroreflector [13] and is not considered. It is seen that the pulse front is overcompensated, the outer part is delayed with respect to the middle one. Since in convex lenses the middle part is delayed more $[13,17]$, a proper choice of the focal length of the focussing lens (Fig. 1b) can achieve exact compensation of the pulse front curvature at the target.

Using the refractive index data of $[18,19]$ for quartz and LiF, respectively, a square coefficient

$\alpha=0.612 \mathrm{fs} / \mathrm{mm}^{2}$

was calculated for the pulse front distortion of the system, just before the focussing lens. The calculated value is significantly different from the experimental one, which would cause more than $200 \mathrm{fs}$ broadening of the pulse at the target (leaving the focussing lens unchanged). A possible explanation of this discrepancy is given in the following.

The basic idea of the pulse front compensation used in this system (Fig. 1) is to use only one compensation element against the distortion along the total optical path. The advantage of this approach is that one has to use only one "overcompensated" achromat instead of using achromats at the position of each lens. However, a disadvantage is that compensation is dependent on the beam diameters at each component. The net pulse front distortion of the system $(\Delta T)$ using a beam diameter $D$ at a lens instead of $D_{0}$ (which the system is corrected for) can be calculated from the distortion $T_{0}$ of the lens with $D_{0}$ as

$\Delta T=T_{0}\left[1-\left(\frac{D_{0}}{D}\right)^{2}\right]$.

The net distortion in our case, when changing the beam diameter from $D_{0}=50 \mathrm{~mm}$ to $D=70 \mathrm{~mm}$ at the last focussing lens ( $\left.T_{0}=380 \mathrm{fs}\right)$ is $\Delta T=190 \mathrm{fs}$. This means that for different beam diameters, the correction or the focal length of the lens must be different.

The other uncertainty of the shape of the pulse front is related to the uncertainty of the refractive index and group velocity index data of the optical materials at this wavelength. Using two different sources $[19,20]$ for the refractive index of $\mathrm{LiF}$ for the design of the achromat lens of the system, a difference of $\Delta T=137 \mathrm{fs}$ is obtained for the distortion of the pulse front. The above considerations show that exact compensation of the system cannot be determined by calculation, but 
must be adjusted experimentally by changing the beam diameter at certain points of the system, say at the last focussing lens. The problem, of course, can be avoided by the use of reflective optics. Such a system - using only reflective optical components - is under construction.

\section{Conclusion}

In conclusion, we have built a pulse-front and pulseduration compensated $\mathrm{KrF}$ laser system, providing $150 \mathrm{fs}, 45 \mathrm{~mJ}$ pulses at the position of the target.

Pulse compression was tested under different experimental conditions. We found that $\sim 150$ fs pulses can be obtained when the pulse compressor is used after the amplifier chain. A single prism pulse compressor is developed allowing high overall transmission.

The optical system is designed to have no overall pulse front distortion. This was checked by measuring the spatial dependence of the pulse front before the last focussing lens.

We improved the construction of the autocorrelators used for the pulse duration and pulse front distortion measurements, to be well suited for high power large aperture beams. The observations are now used in the construction of a high-power $\mathrm{KrF}$ laser system based on reflective optics.

Acknowledgements. The authors wish to thank W. Sauermann for the precise manufacture of the optical components, Dr. N. Ernsting for his contribution to the design of the autocorrelator, and Prof. R. Fedosejevs for his useful comments. This work has been supported by the "Bundesministerium für Forschung und Technologie" and the "Deutsche Forschungsgemeinschaft" by the Gottfried-Wilhelm-Leibniz-Program.

\section{References}

1. S. Szatmári, F.P. Schäfer, E. Müller-Horsche, W. Mückenheim: Opt. Commun. 63, 305 (1987)

2. S. Szatmári, F.P. Schäfer: Opt. Commun. 68, 196 (1988)

3. S. Szatmári, F.P. Schäfer: J. Opt. Soc. Am. B 4, 1943 (1987)

4. A.J. Taylor, R.B. Gibson, J.P. Roberts: Appl. Phys. Lett. 52, 773 (1988)

5. A.P. Schwarzenbach, T.S. Luk, I.A. McIntyre, U. Johann, A. McPherson, K. Boyer, C.K. Rhodes: Opt. Lett. 11, 499 (1986)

6. J.P. Roberts, A.J. Taylor, P.H.Y. Lee, R.B. Gibson: Opt. Lett. 13, 734 (1988)

7. M. Watanabe, A. Endoh, N. Sarukura, S. Watanabe: In Ultrafast Phenomena VI, ed. by T. Yajima, K. Yoshihara, C.B. Harris, S. Shionoya, Springer Ser. Chem. Phys. 48 (Springer, Berlin, Heidelberg 1988) p. 87

8. J.R.M. Barr, N.J. Everall, C.J. Hooker, I.N. Ross, M.J. Shaw, W.T. Toner: Opt. Commun. 66, 127 (1988)

9. W. Tighe, C.H. Nam, J. Robinson, S. Suckever: Rev. Sci. Instrum. 59, 2235 (1988)

10. S. Szatmári: Opt. Quant. Electron. 21, 55 (1989)

11. S. Szatmári, F.P. Schäfer: Appl. Phys. B 46, 305 (1988)

12. A.J. Taylor, R.B. Gibson, J.P. Roberts: Opt. Lett. 13, 814 (1988)

13. S. Szatmári, G, Kühnle: Opt. Commun. 69, 60 (1988)

14. R. Fedosejevs, R. Ottmann, R. Sigel, G. Kühnle, F.P. Schäfer, S. Szatmári: Verhandlungen der Deutschen Physikalischen Gesellschaft 2/1989, DPG-Frühjahrstagung Essen 1989, P 5.1

15. S. Szatmári, F.P. Schäfer: The International Congress on Optical Science and Engineering, 19-23 Sept. 1988, Hamburg, SPIE Technical Abstract 99 (1988)

16. N.P. Ernsting: Private communication

17. Z. Bor: Opt. Lett. 14, 119 (1989)

18. I.M. Malitson: J. Opt. Soc. Am. 55, 1205 (1965)

19. E.D. Palik, W.R. Hunter: In Handbook of Optical Constants of Solids, ed. by E.D. Palik (Academic, New York 1985) p. 675

20. Handbook of Optics, ed. by W.G. Driscoll (McGraw-Hill, New York 1978) pp. 7-95 